\title{
Line graphs and geodesic transitivity ${ }^{*}$
}

\author{
Alice Devillers, Wei Jin, Cai Heng Li , Cheryl E. Praeger \\ Centre for the Mathematics of Symmetry and Computation, School of Mathematics and \\ Statistics, The University of Western Australia, Crawley, WA 6009, Australia
}

Received 21 October 2011, accepted 28 March 2012, published online 1 June 2012

\begin{abstract}
For a graph $\Gamma$, a positive integer $s$ and a subgroup $G \leq \operatorname{Aut}(\Gamma)$, we prove that $G$ is transitive on the set of $s$-arcs of $\Gamma$ if and only if $\Gamma$ has girth at least $2(s-1)$ and $G$ is transitive on the set of $(s-1)$-geodesics of its line graph. As applications, we first classify 2-geodesic transitive graphs of valency 4 and girth 3 , and determine which of them are geodesic transitive. Secondly we prove that the only non-complete locally cyclic 2geodesic transitive graphs are the octahedron and the icosahedron.
\end{abstract}

Keywords: Line graphs, s-geodesic transitive graphs, s-arc transitive graphs.

Math. Subj. Class.: 05E18, 20B25

\section{Introduction}

A geodesic from a vertex $u$ to a vertex $v$ in a graph is a path of shortest length from $u$ to $v$. In the infinite setting geodesics play an important role, for example, in the classification of infinite distance transitive graphs [11], and in studying locally finite graphs, see for example, [17]. They are also used to model, in a finite network, the notion of visibility in Euclidean space [22]. Here we study transitivity properties on geodesics in finite graphs. Throughout this paper, we assume that all graphs are finite simple and undirected.

Let $\Gamma$ be a connected graph with vertex set $V(\Gamma)$, edge set $E(\Gamma)$ and automorphism group $\operatorname{Aut}(\Gamma)$. For a positive integer $s$, an $s$-arc of $\Gamma$ is an $(s+1)$-tuple $\left(v_{0}, v_{1}, \ldots, v_{s}\right)$ of vertices such that $v_{i}, v_{i+1}$ are adjacent and $v_{j-1} \neq v_{j+1}$ for $0 \leq i \leq s-1,1 \leq j \leq s-1$; it is an $s$-geodesic if in addition $v_{0}, v_{s}$ are at distance $s$. For $G \leq \operatorname{Aut}(\Gamma), \Gamma$ is said to be $(G, s)$-arc transitive or $(G, s)$-geodesic transitive, if $\Gamma$ contains an $s$-arc or $s$-geodesic,

\footnotetext{
* This paper forms part of Australian Research Council Federation Fellowship FF0776186 held by the fourth author. The first author is supported by UWA as part of the Federation Fellowship project during most of the work for this paper. The second author is supported by the Scholarships for International Research Fees (SIRF) at UWA.

E-mail addresses: alice.devillers@uwa.edu.au (Alice Devillers),20535692@student.uwa.edu.au (Wei Jin), cai.heng.li@uwa.edu.au (Cai Heng Li), cheryl.praeger@uwa.edu.au (Cheryl E. Praeger)
} 
and $G$ is transitive on the set of $t$-arcs or $t$-geodesics respectively for all $t \leq s$. Moreover, if $G=\operatorname{Aut}(\Gamma)$, then $G$ is usually omitted in the previous notation. The study of $(G, s)$-arc transitive graphs goes back to Tutte's papers $[18,19]$ which showed that if $\Gamma$ is a $(G, s)$-arc transitive cubic graph then $s \leq 5$. About twenty years later, relying on the classification of finite simple groups, Weiss [21] proved that there are no $(G, 8)$-arc transitive graphs with valency at least three. The family of $s$-arc transitive graphs has been studied extensively, see $[2,9,15,16,20]$. Here we consider these properties for line graphs.

The line graph $L(\Gamma)$ of a graph $\Gamma$ is the graph whose vertices are the edges of $\Gamma$, with two edges adjacent in $L(\Gamma)$ if they have a vertex in common. Our first aim in the paper is to investigate connections between the $s$-arc transitivity of a connected graph $\Gamma$ and the $(s-1)$-geodesic transitivity of its line graph $L(\Gamma)$ where $s \geq 2$. A key ingredient in this study is a collection of injective maps $\mathcal{L}_{s}$, where $\mathcal{L}_{s}$ maps the $s$-arcs of $\Gamma$ to certain $s$-tuples of edges of $\Gamma$ (vertices of $L(\Gamma)$ ) as defined in Definition 2.3. The major properties of $\mathcal{L}_{s}$ are derived in Theorem 2.4 and the main consequence linking the symmetry of $\Gamma$ and $L(\Gamma)$ is given in Theorem 1.1, which is proved in Subsection 2.2.

We denote by $\Gamma(u)$ the set of vertices adjacent to the vertex $u$ in $\Gamma$. If $|\Gamma(u)|$ is independent of $u \in V(\Gamma)$, then $\Gamma$ is said to be regular. The girth of $\Gamma$ is the length of the shortest cycle; the diameter diam $(\Gamma)$ of $\Gamma$ is the maximum distance between two vertices.

Theorem 1.1. Let $\Gamma$ be a connected regular, non-complete graph of girth $\mathrm{g}$ and valency at least 3 . Let $G \leq \operatorname{Aut}(\Gamma)$ and let $s$ be a positive integer such that $2 \leq s \leq \operatorname{diam}(L(\Gamma))+1$. Then $G$ is transitive on the set of s-arcs of $\Gamma$ if and only if $s \leq \mathrm{g} / 2+1$ and $G$ is transitive on the set of $(s-1)$-geodesics of $L(\Gamma)$.

It follows from a deep theorem of Richard Weiss in [21] that if $\Gamma$ is a connected $s$-arc transitive graph of valency at least 3 , then $s \leq 7$. This observation yields the following corollary, and its proof can be found in Subsection 2.2.

Corollary 1.2. Let $\Gamma$ and $\mathrm{g}$ be as in Theorem 1.1. Let $s$ be a positive integer such that $2 \leq s \leq \operatorname{diam}(L(\Gamma))+1$. If $L(\Gamma)$ is $(s-1)$-geodesic transitive, then either $2 \leq s \leq 7$ or $s>\max \{7, \mathrm{~g} / 2+1\}$.

Note that in a graph, 1-arcs and 1-geodesics are the same, and are called arcs. For graphs of girth at least 4, each 2-arc is a 2-geodesic so the sets of 2-arc transitive graphs and 2-geodesic transitive graphs are the same. However, there are also 2-geodesic transitive graphs of girth 3 . For such a graph $\Gamma$, the subgraph $[\Gamma(u)]$ induced on the set $\Gamma(u)$ is vertex transitive and contains edges. Moreover, if $[\Gamma(u)]$ is complete, then so is $\Gamma$. A vertex transitive, non-complete, non-empty graph must have at least 4 vertices and thus valency 4 is the first interesting case.

As an application of Theorem 1.1, we characterise connected non-complete 2-geodesic transitive graphs of girth 3 and valency 4 . In this case, $[\Gamma(u)] \cong C_{4}$ or $2 K_{2}$ for each $u \in V(\Gamma)$. If $\Gamma$ is $s$-geodesic transitive with $s=\operatorname{diam}(\Gamma)$, then $\Gamma$ is called geodesic transitive. A graph $\Gamma$ is said to be distance transitive if its automorphism group is transitive on the ordered pairs of vertices at any given distance.

Theorem 1.3. Let $\Gamma$ be a connected non-complete graph of girth 3 and valency 4 . Then $\Gamma$ is 2-geodesic transitive if and only if $\Gamma$ is either $L\left(K_{4}\right) \cong \mathcal{O}$ or $L(\Sigma)$ for a connected 3-arc transitive cubic graph $\Sigma$. 
Moreover, $\Gamma$ is geodesic transitive if and only if $\Gamma=L(\Sigma)$ for a cubic distance transitive graph $\Sigma$, namely $\Sigma=K_{4}, K_{3,3}$, the Petersen graph, the Heawood graph or Tutte's 8-cage.

Since there are infinitely many 3-arc transitive cubic graphs, there are therefore infinitely many 2-geodesic transitive graphs with girth 3 and valency 4 . Theorem 1.3 is proved in Section 3, and it provides a useful method for constructing 2-geodesic transitive graphs of girth 3 and valency 4 which are not geodesic transitive, an example being the line graph of a triple cover of Tutte's 8-cage constructed in [14]. The line graphs mentioned in the second part of Theorem 1.3 are precisely the distance transitive graphs of valency 4 and girth 3 given, for example, in [4, Theorem 7.5 .3 (i)].

A graph $\Gamma$ is said to be locally cyclic if $[\Gamma(u)]$ is a cycle for every vertex $u$. In particular, the girth of a locally cyclic graph is 3 . It was shown in [8, Theorem 1.1] that for 2-geodesic transitive graphs $\Gamma$ of girth 3 , the subgraph $[\Gamma(u)]$ is either a connected graph of diameter 2 , or isomorphic to the disjoint union $m K_{r}$ of $m$ copies of a complete graph $K_{r}$ with $m \geq 2, r \geq 2$. Thus one consequence of Theorem 1.3 is a classification of connected, locally cyclic, 2-geodesic transitive graphs in Corollary 1.4: for $[\Gamma(u)] \cong C_{n}$ has diameter 2 only for valencies $n=4$ or 5, and the valency 5, girth 3,2-geodesic transitive graphs were classified in [7]. Its proof can be found at the end of Section 3. We note that locally cyclic graphs are important for studying embeddings of graphs in surfaces, see for example $[10,12,13]$.

Corollary 1.4. Let $\Gamma$ be a connected, non-complete, locally cyclic graph. Then $\Gamma$ is 2geodesic transitive if and only if $\Gamma$ is the octahedron or the icosahedron.

\section{Line graphs}

We begin by citing a well-known result about line graphs.

Lemma 2.1. [1, p.1455] Let $\Gamma$ be a connected graph. If $\Gamma$ has at least 5 vertices, then $\operatorname{Aut}(\Gamma) \cong \operatorname{Aut}(L(\Gamma))$.

The subdivision graph $S(\Gamma)$ of a graph $\Gamma$ is the graph with vertex set $V(\Gamma) \cup E(\Gamma)$ and edge set $\{\{u, e\} \mid u \in V(\Gamma), e \in E(\Gamma), u \in e\}$. The link between the diameters of $\Gamma$ and $S(\Gamma)$ was determined in [6, Remark 3.1 (b)]: $\operatorname{diam}(S(\Gamma))=2 \operatorname{diam}(\Gamma)+\delta$ for some $\delta \in\{0,1,2\}$. Here, based on this result, we will show the connection between the diameters of $\Gamma$ and $L(\Gamma)$ in the following lemma.

Lemma 2.2. Let $\Gamma$ be a connected graph with $|V(\Gamma)| \geq 2$. Then it holds $\operatorname{diam}(L(\Gamma))=$ $\operatorname{diam}(\Gamma)+x$ for some $x \in\{-1,0,1\}$. Moreover, all three values occur, for example, if $\Gamma=K_{3+x}$, then $\operatorname{diam}(L(\Gamma))=\operatorname{diam}(\Gamma)+x=1+x$ for each $x$.

Proof. Let $d=\operatorname{diam}(\Gamma), d_{l}=\operatorname{diam}(L(\Gamma))$ and $d_{s}=\operatorname{diam}(S(\Gamma))$. Let $\left(x_{0}, x_{2}, \ldots, x_{2 d_{l}}\right)$ be a $d_{l}$-geodesic of $L(\Gamma)$. Then by definition of $L(\Gamma)$, each edge intersection $x_{2 i} \cap x_{2 i+2}$ is a vertex $v_{2 i+1}$ of $\Gamma$ and $\left(x_{0}, v_{1}, x_{2}, \ldots, v_{2 d_{l}-1}, x_{2 d_{l}}\right)$ is a $2 d_{l}$-path in $S(\Gamma)$. Suppose that $\left(x_{0}, v_{1}, x_{2}, \ldots, v_{2 d_{l}-1}, x_{2 d_{l}}\right)$ is not a $2 d_{l}$-geodesic of $S(\Gamma)$. Then there is an $r$-geodesic from $x_{0}$ to $x_{2 d_{l}}$, say $\left(y_{0}, y_{1}, y_{2}, \ldots, y_{r}\right)$ with $y_{0}=x_{0}$ and $y_{r}=x_{2 d_{l}}$, such that $r<2 d_{l}$. Since both $x_{0}, x_{2 d_{l}}$ are in $V(L(\Gamma))$, it follows that $r$ is even, and hence $d_{L(\Gamma)}\left(x_{0}, x_{2 d_{l}}\right)=$ $\frac{r}{2}<d_{l}$ which contradicts the fact that $\left(x_{0}, x_{2}, \ldots, x_{2 d_{l}}\right)$ is a $d_{l}$-geodesic of $L(\Gamma)$. Thus 
$\left(x_{0}, v_{1}, x_{2}, \ldots, v_{2 d_{l}-1}, x_{2 d_{l}}\right)$ is a $2 d_{l}$-geodesic in $S(\Gamma)$. It follows from [6, Remark 3.1 (b)] that $d_{l} \leq d_{s} / 2 \leq d+1$.

Now take a $d_{s}$-geodesic $\left(x_{0}, x_{1}, \ldots, x_{d_{s}}\right)$ in $S(\Gamma)$. If $x_{0} \in E(\Gamma)$, then $\left(x_{0}, x_{2}, x_{4}, \ldots\right.$, $\left.x_{2\left\lfloor d_{s} / 2\right\rfloor}\right)$ is a $\left\lfloor d_{s} / 2\right\rfloor$-geodesic in $L(\Gamma)$, so $d_{l} \geq\left\lfloor d_{s} / 2\right\rfloor \geq d$. Similarly we see that $d_{l} \geq d$ if $x_{d_{s}} \in E(\Gamma)$. Finally if both $x_{0}, x_{d_{s}} \in V(\Gamma)$, then $d_{s}$ is even and $d_{\Gamma}\left(x_{0}, x_{d_{s}}\right)=d_{s} / 2$. Moreover $\left(x_{1}, x_{3}, \ldots, x_{d_{s}-1}\right)$ is a $\left(\frac{d_{s}-2}{2}\right)$-geodesic in $L(\Gamma)$. By [6, Remark $\left.3.1(\mathrm{~b})\right], d_{s}=$ $2 d$, so $d_{l} \geq \frac{d_{s}-2}{2}=d-1$.

\subsection{The map $\mathcal{L}_{s}$}

Let $\Gamma$ be a finite connected graph. For each integer $s \geq 2$, we define a map from the set of $s$-arcs of $\Gamma$ to the set of $s$-tuples of $V(L(\Gamma))$.

Definition 2.3. Let $\mathbf{a}=\left(v_{0}, v_{1}, \ldots, v_{s}\right)$ be an $s$-arc of $\Gamma$ where $s \geq 2$, and for $0 \leq i<s$, let $e_{i}:=\left\{v_{i}, v_{i+1}\right\} \in E(\Gamma)$. Define $\mathcal{L}_{s}(\mathbf{a}):=\left(e_{0}, e_{1}, \ldots, e_{s-1}\right)$.

The following theorem gives some important properties of $\mathcal{L}_{s}$.

Theorem 2.4. Let $s \geq 2$, let $\Gamma$ be a connected graph containing at least one s-arc, and let $\mathcal{L}_{s}$ be as in Definition 2.3. Then the following statements hold.

(1) $\mathcal{L}_{s}$ is an injective map from the set of $s$-arcs of $\Gamma$ to the set of $(s-1)$-arcs of $L(\Gamma)$. Further, $\mathcal{L}_{s}$ is a bijection if and only if either $s=2$, or $s \geq 3$ and $\Gamma \cong C_{m}$ or $P_{n}$ for some $m \geq 3, n \geq s$.

(2) $\mathcal{L}_{s}$ maps $s$-geodesics of $\Gamma$ to $(s-1)$-geodesics of $L(\Gamma)$.

(3) If $s \leq \operatorname{diam}(L(\Gamma))+1$, then the image $\operatorname{Im}\left(\mathcal{L}_{s}\right)$ contains the set $\mathcal{G}_{s-1}$ of all $(s-1)$ geodesics of $L(\Gamma)$. Moreover, $\operatorname{Im}\left(\mathcal{L}_{s}\right)=\mathcal{G}_{s-1}$ if and only if $\operatorname{girth}(\Gamma) \geq 2 s-2$.

(4) $\mathcal{L}_{s}$ is Aut $(\Gamma)$-equivariant, that is, $\mathcal{L}_{s}(\boldsymbol{a})^{g}=\mathcal{L}_{s}\left(\boldsymbol{a}^{g}\right)$ for all $g \in \operatorname{Aut}(\Gamma)$ and all $s-\operatorname{arcs} \boldsymbol{a}$ of $\Gamma$.

Proof. (1) Let $\mathbf{a}=\left(v_{0}, v_{1}, \ldots, v_{s}\right)$ be an $s$-arc of $\Gamma$ and let $\mathcal{L}_{s}(\mathbf{a}):=\left(e_{0}, e_{1}, \ldots, e_{s-1}\right)$ with the $e_{i}$ as in Definition 2.3. Then each of the $e_{i}$ lies in $E(\Gamma)=V(L(\Gamma))$ and $e_{k} \neq e_{k+1}$ for $0 \leq k \leq s-2$. Further, since $v_{j} \neq v_{j+1}, v_{j+2}$ for $1 \leq j \leq s-2$, we have $e_{j-1} \neq e_{j+1}$. Thus $\mathcal{L}_{s}(\mathbf{a})$ is an $(s-1)$-arc of $L(\Gamma)$.

Let $\mathbf{b}=\left(u_{0}, u_{1}, \ldots, u_{s}\right)$ and $\mathbf{c}=\left(w_{0}, w_{1}, \ldots, w_{s}\right)$ be two $s$-arcs of $\Gamma$. Then $\mathcal{L}_{s}(\mathbf{b})=$ $\left(f_{0}, f_{1}, \ldots, f_{s-1}\right)$ and $\mathcal{L}_{s}(\mathbf{c})=\left(g_{0}, g_{1}, \ldots, g_{s-1}\right)$ are two $(s-1)$-arcs of $L(\Gamma)$, where $f_{i}=\left\{u_{i}, u_{i+1}\right\}$ and $g_{i}=\left\{w_{i}, w_{i+1}\right\}$ for $0 \leq i<s$. Suppose that $\mathcal{L}_{s}(\mathbf{b})=\mathcal{L}_{s}(\mathbf{c})$. Then $f_{i}=g_{i}$ for each $i \geq 0$, and hence $f_{i} \cap f_{i+1}=g_{i} \cap g_{i+1}$, that is, $u_{i+1}=w_{i+1}$ for each $0 \leq i \leq s-2$. So also $u_{0}=w_{0}$ and $u_{s}=w_{s}$, and hence $\mathbf{b}=\mathbf{c}$. Thus $\mathcal{L}_{s}$ is injective.

Now we prove the second part. Each arc of $L(\Gamma)$ is of the form $\mathbf{h}=(e, f)$ where $e=\left\{u_{0}, u_{1}\right\}$ and $f=\left\{u_{1}, u_{2}\right\}$ are distinct edges of $\Gamma$. Thus $u_{0} \neq u_{2}$, so $\mathbf{k}=\left(u_{0}, u_{1}, u_{2}\right)$ is a 2 -arc of $\Gamma$ and $\mathcal{L}_{2}(\mathbf{k})=\mathbf{h}$. It follows that $\mathcal{L}_{2}$ is onto and hence is a bijection. If $s \geq 3$ and $\Gamma \cong C_{m}$ or $P_{n}$ for some $m \geq 3, n \geq s$, then $L(\Gamma) \cong C_{m}$ or $P_{n-1}$ respectively, and hence for every $(s-1)$-arc $\mathbf{x}$ of $L(\Gamma)$, we can find an $s$-arc $\mathbf{y}$ of $\Gamma$ such that $\mathcal{L}_{s}(\mathbf{y})=\mathbf{x}$, that is, $\mathcal{L}_{s}$ is onto. Thus $\mathcal{L}_{s}$ is a bijection. Conversely, suppose that $\mathcal{L}_{s}$ is onto, and that $s \geq 3$. Assume that some vertex $u$ of $\Gamma$ has valency greater than 2 and let $e_{1}=\left\{u, v_{1}\right\}, e_{2}=$ $\left\{u, v_{2}\right\}, e_{3}=\left\{u, v_{3}\right\}$ be distinct edges. Then $\mathbf{x}=\left(e_{1}, e_{2}, e_{3}\right)$ is a 2-arc in $L(\Gamma)$ and there is no 3 -arc $\mathbf{y}$ of $\Gamma$ such that $\mathcal{L}_{s}(\mathbf{y})=\mathbf{x}$. In general, for $s=3 a+b \geq 4$ with $a \geq 1$ and $b \in\{0,1,2\}$, we concatenate $a$ copies of $\mathbf{x}$ to form an $(s-1)$-arc of $L(\Gamma)$ : namely $\left(\mathbf{x}^{a}\right)$ if $b=0 ;\left(\mathbf{x}^{a}, e_{1}\right)$ if $b=1 ;\left(\mathbf{x}^{a}, e_{1}, e_{2}\right)$ if $b=2$. This $(s-1)$-arc does not lie in the image 
of $\mathcal{L}_{s}$. Thus each vertex of $\Gamma$ has valency at most 2 . If all vertices have valency 2 then $\Gamma \cong C_{m}$ for some $m \geq 3$, since $\Gamma$ is connected. So suppose that some vertex $u$ of $\Gamma$ has valency 1 . Since $\Gamma$ is connected and each other vertex has valency at most 2 , it follows that $\Gamma \cong P_{n}$ for some $n \geq s$.

(2) Let $\mathbf{a}=\left(v_{0}, \ldots, v_{s}\right)$ be an $s$-geodesic of $\Gamma$ and let $\mathcal{L}_{s}(\mathbf{a})=\left(e_{0}, \ldots, e_{s-1}\right)$ as above. If $s=2$, then $\mathcal{L}_{s}(\mathbf{a})$ is a 1-arc, and hence a 1-geodesic of $L(\Gamma)$. Suppose that $s \geq 3$ and $\mathcal{L}_{s}(\mathbf{a})$ is not an $(s-1)$-geodesic. Then $d_{L(\Gamma)}\left(e_{0}, e_{s-1}\right)=r<s-1$ and there exists an $r$-geodesic $\mathbf{r}=\left(f_{0}, f_{1}, \ldots, f_{r-1}, f_{r}\right)$ with $f_{0}=e_{0}$ and $f_{r}=e_{s-1}$. Since $s \geq 3$ and $\mathbf{a}$ is an $s$-geodesic, it follows that $\left\{v_{0}, v_{1}\right\} \cap\left\{v_{s-1}, v_{s}\right\}=\emptyset$, that is, $e_{0}$ and $e_{s-1}$ are not adjacent in $L(\Gamma)$. Thus $r \geq 2$. Since $\mathbf{r}$ is an $r$-geodesic, it follows that the consecutive edges $f_{i-1}, f_{i}, f_{i+1}$ do not share a common vertex for any $1 \leq i \leq r-1$, otherwise $\left(f_{0}, \ldots, f_{i-1}, f_{i+1}, \ldots, f_{r}\right)$ would be a shorter path than $\mathbf{r}$, which is impossible. Hence we have $f_{h}=\left\{u_{h}, u_{h+1}\right\}$ for $0 \leq h \leq r$. Then $\left(u_{1}, u_{2}, \ldots, u_{r}\right)$ is an $(r-1)$-path in $\Gamma,\left\{u_{1}\right\}=e_{0} \cap f_{1} \subseteq\left\{v_{0}, v_{1}\right\}$ and $\left\{u_{r}\right\}=f_{r-1} \cap e_{s-1} \subseteq\left\{v_{s-1}, v_{s}\right\}$. It follows that $d_{\Gamma}\left(v_{0}, v_{s}\right) \leq d_{\Gamma}\left(u_{1}, u_{r}\right)+2 \leq r+1<s$, contradicting the fact that $\mathbf{a}$ is an $s$-geodesic. Therefore, $\mathcal{L}_{s}(\mathbf{a})$ is an $(s-1)$-geodesic of $L(\Gamma)$.

(3) Let $2 \leq s \leq \operatorname{diam}(L(\Gamma))+1$ and $\mathcal{G}_{s-1}$ be the set of all $(s-1)$-geodesics of $L(\Gamma)$. If $s=2$, then by part (1), each 1 -geodesic of $L(\Gamma)$ lies in the image $\operatorname{Im}\left(\mathcal{L}_{2}\right)$, and hence $\mathcal{G}_{1} \subseteq \operatorname{Im}\left(\mathcal{L}_{2}\right)$. Now suppose inductively that $2 \leq s \leq \operatorname{diam}(L(\Gamma))$ and $\mathcal{G}_{s-1} \subseteq \operatorname{Im}\left(\mathcal{L}_{s}\right)$. Let $\mathbf{e}=\left(e_{0}, e_{1}, e_{2}, \ldots, e_{s}\right)$ be an $s$-geodesic of $L(\Gamma)$. Then $\mathbf{e}^{\prime}=\left(e_{0}, e_{1}, e_{2}, \ldots, e_{s-1}\right)$ is an $(s-1)$-geodesic of $L(\Gamma)$. Thus there exists an $s$-arc a of $\Gamma$ such that $\mathcal{L}_{s}(\mathbf{a})=\mathbf{e}^{\prime}$, say $\mathbf{a}=\left(v_{0}, v_{1}, \ldots, v_{s}\right)$. Since $e_{s}$ is adjacent to $e_{s-1}=\left\{v_{s-1}, v_{s}\right\}$ but not to $e_{s-2}=$ $\left\{v_{s-2}, v_{s-1}\right\}$ in $L(\Gamma)$, it follows that $e_{s}=\left\{v_{s}, x\right\}$ where $x \notin\left\{v_{s-2}, v_{s-1}\right\}$. Hence $\mathbf{b}=$ $\left(v_{0}, v_{1}, \ldots, v_{s}, x\right)$ is an $(s+1)$-arc of $\Gamma$. Further, $\mathcal{L}_{s+1}(\mathbf{b})=\mathbf{e}$. Thus $\operatorname{Im}\left(\mathcal{L}_{s+1}\right)$ contains all $s$-geodesics of $L(\Gamma)$, that is, $\mathcal{G}_{s} \subseteq \operatorname{Im}\left(\mathcal{L}_{s+1}\right)$. Hence the first part of (3) is proved by induction.

Now we prove the second part. Suppose first that for every $s$-arc a of $\Gamma, \mathcal{L}_{s}(\mathbf{a})$ is an $(s-1)$-geodesic of $L(\Gamma)$. Let $\mathrm{g}:=\operatorname{girth}(\Gamma)$. If $s=2$, as $\mathrm{g} \geq 3$, then $\mathrm{g} \geq 2 s-2$. Now let $s \geq 3$. Assume that $\mathrm{g} \leq 2 s-3$. Then $\Gamma$ has a g-cycle $\mathbf{b}=\left(u_{0}, u_{1}, u_{2}, \ldots, u_{\mathrm{g}-1}, u_{\mathrm{g}}\right)$ with $u_{\mathrm{g}}=u_{0}$. It follows that $\mathcal{L}_{\mathrm{g}}(\mathbf{b})$ forms a g-cycle of $L(\Gamma)$. Thus the sequence $\mathbf{b}^{\prime}=$ $\left(u_{0}, u_{1}, \ldots, u_{s}\right)$ (where we take subscripts modulo $g$ if necessary) is an $s$-arc of $\Gamma$ and $\mathcal{L}_{s}\left(\mathbf{b}^{\prime}\right)=\left(e_{0}, e_{1}, \ldots, e_{s-1}\right)$ involves only the vertices of $\mathcal{L}_{s}(\mathbf{b})$. This implies that $d_{L(\Gamma)}\left(e_{0}, e_{s-1}\right) \leq \frac{\mathrm{g}}{2} \leq \frac{2 s-3}{2}<s-1$, that is, $\mathcal{L}_{s}\left(\mathbf{b}^{\prime}\right)$ is not an $(s-1)$-geodesic, which is a contradiction. Thus, $\mathrm{g} \geq 2 s-2$.

Conversely, suppose that $\mathrm{g} \geq 2 s-2$. Let $\mathbf{a}:=\left(v_{0}, v_{1}, v_{2}, \ldots, v_{s}\right)$ be an $s$-arc of $\Gamma$. Then $\mathcal{L}_{s}(\mathbf{a})=\left(e_{0}, e_{1}, e_{2}, \ldots, e_{s-1}\right)$ is an $(s-1)$-arc of $L(\Gamma)$ by part (1). Let $\mathbf{a}^{\prime}:=$ $\left(v_{0}, v_{1}, v_{2}, \ldots, v_{s-1}\right)$. Since $\mathrm{g} \geq 2 s-2$, it follows that $\mathbf{a}^{\prime}$ is an $(s-1)$-geodesic, and hence by (2), $\mathcal{L}_{s-1}\left(\mathbf{a}^{\prime}\right)=\left(e_{0}, e_{1}, e_{2}, \ldots, e_{s-2}\right)$ is an $(s-2)$-geodesic of $L(\Gamma)$. Thus $z=d_{L(\Gamma)}\left(e_{0}, e_{s-1}\right)$ satisfies $s-3 \leq z \leq s-1$. There is a $z$-geodesic from $e_{0}$ to $e_{s-1}$, say $\mathbf{f}=\left(e_{0}, f_{1}, f_{2}, \ldots, f_{z-1}, e_{s-1}\right)$. Further, by the first part of (3), there is a $(z+1)$-arc $\mathbf{b}=$ $\left(u_{0}, u_{1}, \ldots, u_{z}, u_{z+1}\right)$ of $\Gamma$ such that $\mathcal{L}_{z+1}(\mathbf{b})=\mathbf{f}$ and we have $e_{0}=\left\{u_{0}, u_{1}\right\}=\left\{v_{0}, v_{1}\right\}$ and $e_{s-1}=\left\{u_{z}, u_{z+1}\right\}=\left\{v_{s-1}, v_{s}\right\}$. There are 4 cases, in columns 2 and 3 of Table 1: in each case there is a given nondegenerate closed walk $\mathbf{x}$ of length $l(\mathbf{x})$ as in Table 1 . Thus $l(\mathbf{x}) \geq \mathrm{g} \geq 2 s-2$ and in each case $l(\mathbf{x}) \leq s+z-1$. It follows that $z \geq s-1$, and hence $z=s-1$. Thus $\mathcal{L}_{s}(\mathbf{a})=\left(e_{0}, e_{1}, e_{2}, \ldots, e_{s-1}\right)$ is an $(s-1)$-geodesic of $L(\Gamma)$.

(4) This property follows from the definition of $\mathcal{L}_{s}$. 
Table 1: Four cases of $\mathbf{x}$

\begin{tabular}{|c|c|c|c|c|}
\hline Case & $\left(u_{0}, u_{1}\right)$ & $\left(u_{z}, u_{z+1}\right)$ & $\mathbf{x}$ & $l(\mathbf{x})$ \\
\hline 1 & $\left(v_{0}, v_{1}\right)$ & $\left(v_{s-1}, v_{s}\right)$ & $\left(v_{s-1}, v_{s-2}, \ldots, v_{2}, v_{1}, u_{2}, \ldots\right.$, & $s+z-3$ \\
& & & $\left.u_{z-1}, v_{s-1}\right)$ & \\
\hline 2 & $\left(v_{0}, v_{1}\right)$ & $\left(v_{s}, v_{s-1}\right)$ & $\left(v_{s}, v_{s-1}, \ldots, v_{2}, v_{1}, u_{2}, \ldots\right.$, & $s+z-2$ \\
& & & $\left.u_{z-1}, v_{s}\right)$ & \\
\hline 3 & $\left(v_{1}, v_{0}\right)$ & $\left(v_{s-1}, v_{s}\right)$ & $\left(v_{s-1}, v_{s-2}, \ldots, v_{2}, v_{1}, u_{1}, u_{2}, \ldots\right.$, & $s+z-2$ \\
& & & $\left.u_{z-1}, v_{s-1}\right)$ & \\
\hline 4 & $\left(v_{1}, v_{0}\right)$ & $\left(v_{s}, v_{s-1}\right)$ & $\left(v_{s}, v_{s-1}, \ldots, v_{2}, v_{1}, u_{1}, u_{2}, \ldots\right.$, & $s+z-1$ \\
& & & $\left.u_{z-1}, v_{s}\right)$ & \\
\hline
\end{tabular}

Remark 2.5. (i) The map $\mathcal{L}_{s}$ is usually not surjective on the set of $(s-1)$-arcs of $L(\Gamma)$. In the proof of Theorem $2.4(1)$, we constructed an $(s-1)$-arc of $L(\Gamma) \operatorname{not} \operatorname{in} \operatorname{Im}\left(\mathcal{L}_{s}\right)$ for any $\Gamma$ with at least one vertex of valency at least 3 .

(ii) Theorem 2.4 (1) and (3) imply that, for each $(s-1)$-geodesic e of $L(\Gamma)$, there is a unique $s$-arc a of $\Gamma$ such that $\mathcal{L}_{s}(\mathbf{a})=\mathbf{e}$. The $s$-arc a is not always an $s$-geodesic. For example, if $\Gamma$ has girth 3 and $\left(v_{0}, v_{1}, v_{2}, v_{0}\right)$ is a 3 -cycle, then $\mathbf{a}=\left(v_{0}, v_{1}, v_{2}\right)$ is not a 2-geodesic but $\mathcal{L}_{2}(\mathbf{a})$ is the 1-geodesic $\left(e_{0}, e_{1}\right)$ where $e_{0}=\left\{v_{0}, v_{1}\right\}$ and $e_{1}=\left\{v_{1}, v_{2}\right\}$.

\subsection{Proofs of Theorem 1.1 and Corollary 1.2}

Proof of Theorem 1.1. Let $\Gamma$ be a connected, regular, non-complete graph of girth $g$ and valency at least 3 . Then in particular $|V(\Gamma)| \geq 5$, and by Lemma 2.1 , Aut $(\Gamma) \cong$ $\operatorname{Aut}(L(\Gamma))$. Let $G \leq \operatorname{Aut}(\Gamma)$ and let $2 \leq s \leq \operatorname{diam}(L(\Gamma))+1$.

Suppose first that $G$ is transitive on the set of $s$-arcs of $\Gamma$. Then by [3, Proposition 17.2], $s \leq \mathrm{g} / 2+1$. Since $s-1 \leq \operatorname{diam}(L(\Gamma))$, it follows that $L(\Gamma)$ has $(s-1)$-geodesics and by Theorem $2.4(3), \operatorname{Im}\left(\mathcal{L}_{s}\right)$ is the set of $(s-1)$-geodesics of $L(\Gamma)$. On the other hand, by Theorem 2.4 (4), $G$ acts transitively on $\operatorname{Im}\left(\mathcal{L}_{s}\right)$, and hence $G$ is transitive on the set of $(s-1)$-geodesics of $L(\Gamma)$.

Conversely, suppose that $s \leq \mathrm{g} / 2+1$ and $G$ is transitive on the $(s-1)$-geodesics of $L(\Gamma)$. Then by the last assertion of Theorem $2.4(3), \operatorname{Im}\left(\mathcal{L}_{s}\right)$ is the set of $(s-1)$-geodesics, and since $\mathcal{L}_{s}$ is injective, it follows from Theorem 2.4 (1) and (4) that $G$ is transitive on the set of $s$-arcs of $\Gamma$.

Proof of Corollary 1.2. Let $\Gamma, \mathrm{g}, s$ be as in Theorem 1.1. Assume that Aut $(\Gamma)$ is transitive on the $(s-1)$-geodesics of $L(\Gamma)$. If $s>7$, then by [21], Aut $(\Gamma)$ is not transitive on the $s$-arcs of $\Gamma$ and so by Theorem 1.1, $s>\frac{\mathrm{g}}{2}+1$.

\section{2-geodesic transitive graphs that are locally cyclic or locally $2 K_{2}$}

In this section, we prove Theorem 1.3. The proof uses the notion of a clique graph. A maximum clique of a graph $\Gamma$ is a clique (complete subgraph) which is not contained in a larger clique. The clique graph $C(\Gamma)$ of $\Gamma$ is the graph with vertices the maximum cliques of $\Gamma$, and two maximum cliques are adjacent if and only if they have at least one common vertex in $\Gamma$. 
Proof of Theorem 1.3. Let $\Gamma$ be a connected non-complete graph of girth 3 and valency 4 , and let $A=\operatorname{Aut}(\Gamma)$ and $v \in V(\Gamma)$. Suppose first that $\Gamma$ is 2 -geodesic transitive. Then $\Gamma$ is arc transitive, and so $A_{v}$ is transitive on $\Gamma(v)$. Since $\Gamma$ is non-complete of girth $3,[\Gamma(v)]$ is neither complete nor edgeless, and so, as discussed before the statement of Theorem 1.3, $[\Gamma(v)]=C_{4}$ or $2 K_{2}$. If $[\Gamma(v)] \cong C_{4}$, then it is easy to see that $\Gamma \cong \mathcal{O}$ (or see [4, p.5] or [5]). So we may assume that $[\Gamma(v)] \cong 2 K_{2}$. It follows from [8, Theorem 1.4] that $\Gamma$ is isomorphic to the clique graph $C(\Sigma)$ of a connected graph $\Sigma$ such that, for each $u \in V(\Sigma)$, the induced subgraph $[\Sigma(u)] \cong 3 K_{1}$, that is to say, $\Sigma$ is a cubic graph of girth at least 4 and $C(\Sigma)$ is in this case the line graph $L(\Sigma)$. Moreover, [8, Theorem 1.4] gives that $\Sigma \cong C(\Gamma)$. A cubic graph with girth at least 4 has $|V(\Sigma)| \geq 5$, so by Lemma 2.1, $A \cong \operatorname{Aut}(\Sigma)$. Now we apply Theorem 1.1 to the graph $\Sigma$ of girth $\mathrm{g} \geq 4$. Since $\Gamma=L(\Sigma)$ is 2 -geodesic transitive and $3 \leq \mathrm{g} / 2+1$, it follows from Theorem 1.1 that $\Sigma$ is 3 -arc transitive. Therefore, $\Gamma$ is the line graph of a 3 -arc transitive cubic graph.

Conversely, if $\Gamma \cong \mathcal{O}$, then it is 2 -geodesic transitive, and hence is geodesic transitive as $\operatorname{diam}(\mathcal{O})=2$. If $\Gamma=L(\Sigma)$ where $\Sigma$ is a 3 -arc transitive cubic graph, then by Theorem 1.1 applied to $\Sigma$ with $s=3, L(\Sigma)$ is 2-geodesic transitive. This proves the first assertion of Theorem 1.3.

To prove the second assertion, suppose first that $\Gamma$ is geodesic transitive. Then $\Gamma$ is distance transitive, and so by Theorems 7.5.2 and 7.5.3 (i) of [4], $\Gamma$ is one of the following graphs: $\mathcal{O}=L\left(K_{4}\right), H(2,3)=L\left(K_{3,3}\right)$, or the line graph of the Petersen graph, the Heawood graph or Tutte's 8-cage. We complete the proof by showing that all these graphs are geodesic transitive. As noted above, $\mathcal{O}$ is geodesic transitive; by [7, Proposition 3.2], $H(2,3)$ is geodesic transitive. It remains to consider the last three graphs.

Let $\Sigma$ be the Petersen graph and $\Gamma=L(\Sigma)$. Then $\Sigma$ is 3-arc transitive, and it follows from Theorem 1.1 that $\Gamma$ is 2 -geodesic transitive. By [4, Theorem 7.5.3 (i)], $\operatorname{diam}(\Gamma)=3$ and $\left|\Gamma(w) \cap \Gamma_{3}(u)\right|=1$ for each 2-geodesic $(u, v, w)$ of $\Gamma$. Thus $\Gamma$ is 3-geodesic transitive, and hence is geodesic transitive.

Let $\Sigma_{1}$ be the Heawood graph and $\Sigma_{2}$ be Tutte's 8-cage. Then $\Sigma_{1}$ is 4 -arc transitive and $\Sigma_{2}$ is 5-arc transitive, and hence by Theorem 1.1, $L\left(\Sigma_{1}\right)$ is 3-geodesic transitive and $L\left(\Sigma_{2}\right)$ is 4-geodesic transitive. By [4, Theorem 7.5.3 (i)], $\operatorname{diam}\left(L\left(\Sigma_{1}\right)\right)=3$ and $\operatorname{diam}\left(L\left(\Sigma_{2}\right)\right)=$ 4 , and hence both $L\left(\Sigma_{1}\right)$ and $L\left(\Sigma_{2}\right)$ are geodesic transitive.

Finally, we prove Corollary 1.4.

Proof of Corollary 1.4. Let $\Gamma$ be a connected non-complete locally cyclic graph. If $\Gamma$ is 2 -geodesic transitive, then it is regular of valency $n$ say. As discussed in the introduction, $n=4$ or 5 . If $n=4$, then we proved in Theorem 1.3, that $\Gamma$ is isomorphic to the octahedron and that the octahedron is indeed 2-geodesic transitive. If $n=5$, then by [7, Theorem 1.2], $\Gamma$ is isomorphic to the icosahedron, and this graph is 2-geodesic transitive.

\section{Acknowledgements}

This article forms part of the second author's Ph.D thesis at UWA under the supervision of other authors. The authors are grateful to Sandi Malnič for suggesting that we consider $s$-geodesic transitivity for locally cyclic graphs. They also thank the anonymous referees for valuable suggestions on the exposition. 


\section{References}

[1] L. Babai, Automorphism Groups, Isomorphism, Reconstruction, Handbook of Combinatorics, the MIT Press, Cambridge, Massachusetts, Amsterdam-Lausanne-New York, Vol 2, (1995), 1447-1540.

[2] R. W. Baddeley, Two-arc transitive graphs and twisted wreath products. J. Algebraic Combin. 2 (1993), 215-237.

[3] N. L. Biggs, Algebraic Graph Theory, Second ed., Cambridge University Press, Cambridge, (1993).

[4] A. E. Brouwer, A. M. Cohen and A. Neumaier, Distance-Regular Graphs, Springer Verlag, Berlin, Heidelberg, New York, (1989).

[5] A. M. Cohen, Local recognition of graphs, buildings, and related geometries, in: W. M. Kantor, R. A. Liebler, S. E. Payne, E. E. Shult (eds.), Finite Geometries, Buildings, and related Topics, Oxford Sci. Publ., New York. 19 (1990), 85-94.

[6] A. Daneshkhah, A. Devillers and C. E. Praeger, Symmetry properties of subdivision graphs, Discrete Math. 312 (2012), 86-93.

[7] A. Devillers, W. Jin, C. H. Li and C. E. Praeger, On distance, geodesic and arc transitivity of graphs, preprint, 2011, available at http://arxiv.org/abs/1110.2235.

[8] A. Devillers, W. Jin, C. H. Li and C. E. Praeger, Local 2-geodesic transitivity and clique graphs, in preparation.

[9] A. A. Ivanov and C. E. Praeger, On finite affine 2-arc transitive graphs, European J. Combin. 14 (1993), 421-444.

[10] M. Juvan, A. Malnič and B. Mohar, Systems of curves on surfaces, J. Combin. Theory Ser. B 68 (1996), 7-22.

[11] H. D. Macpherson, Infinite distance transitive graphs of finite valency, Combinatorica 2 (1982), 63-69.

[12] A. Malnič and B. Mohar, Generating locally cyclic triangulations of surfaces, J. Combin. Theory Ser. B 56 (1992), 147-164.

[13] A. Malnič and R. Nedela, K-Minimal triangulations of surfaces, Acta Math. Univ. Comenianae LXIV 1 (1995), 57-76.

[14] M. J. Morton, Classification of 4 and 5-arc transitive cubic graphs of small girth, J. Austral. Math. Soc. A 50 (1991), 138-149.

[15] C. E. Praeger, An O'Nan Scott theorem for finite quasiprimitive permutation groups and an application to 2-arc transitive graphs, J. London Math. Soc. 47 (1993), 227-239.

[16] C. E. Praeger, On a reduction theorem for finite, bipartite, 2-arc transitive graphs, Australas. J. Combin. 7 (1993) 21-36.

[17] C. Thomassen and W. Woess, Vertex-transitive graphs and accessibility, J. Combin. Theory Ser. B 58 (1993), 248-268.

[18] W. T. Tutte, A family of cubical graphs, Proc. Cambridge Philos. Soc. 43 (1947), 459-474.

[19] W. T. Tutte, On the symmetry of cubic graphs, Canad. J. Math. 11 (1959), 621-624.

[20] R. Weiss, s-transitive graphs, Algebraic methods in graph theory, Vol. I, II, (Szeged, 1978), Colloq. Math. Soc. Janos Bolyai, 25, North-Holland, Amsterdam-New York, (1981), 827-847.

[21] R. Weiss, The non-existence of 8-transitive graphs, Combinatorica 1 (1981), 309-311.

[22] A. Y. Wu and A. Rosenfeld, Geodesic visibility in graphs, J. Information Sciences 108 (1998), $5-12$. 